\title{
Hemorrhagic Fever with Renal Syndrome, Zibo City, China, 2006-2014
}

\section{Ling Wang, Tao Wang, Feng Cui, Shen-Yong Zhai, Ling Zhang, Shu-Xia Yang, Zhi-Qiang Wang, Xue-Jie Yu}

Analysis of hemorrhagic fever with renal syndrome cases in Zibo City, China, during 2006-2014 showed that it occurred year-round. Peaks in spring and fall/winter were caused by Hantaan and Seoul viruses, respectively. Rodent hosts were the striped field mouse for Hantaan virus and the brown rat and house mouse for Seoul virus.

$\mathrm{H}$ emorrhagic fever with renal syndrome (HFRS) is caused by hantavirus and transmitted primary by rodents $(1,2)$. HFRS occurs worldwide, but $\approx 90 \%$ of HFRS cases have been reported in China (3). During 2006-2012, a total of 77,558 HFRS cases, including 866 deaths, were reported in China (4). Zibo City in Shandong Province, located in eastern China, has a high incidence rate of HFRS $(5,6)$. We analyzed the clinical data for HFRS cases and surveyed the prevalence of hantaviruses in rodent populations in rural area of Zibo City during 2006-2014.

\section{The Study}

Zibo City is a prefecture-level city located at $36^{\circ} 47^{\prime} \mathrm{N}$ $118^{\circ} 3^{\prime} \mathrm{E}$ (Figure 1). The city consists of 6 districts and 3 counties distributed over $5,938 \mathrm{~km}^{2}$ of land; the total population during the 2010 census was 4.53 million, of whom 900,000 persons were farmers. The city is $\approx 42 \%$ mid-sized mountains in the south, $30 \%$ hills in the center, and $28 \%$ plains in the north (http://115.238.252.51:9001/ html/2007/09/13/20070913141750.html).

We obtained data for HFRS patients from the Zibo Center for Disease Control and Prevention and analyzed these data with Excel 2007 (Microsoft, Redmond, WA, USA) and SPSS 16.0 software (SPSS Inc., Chicago, IL, USA) and $\chi^{2}$ test for statistical analysis. The research protocol was approved by the human bioethics committee of the Zibo Center for Disease Control and Prevention, and all participants provided written informed consent. HFRS cases were confirmed by detection of hantavirus IgM

Author affiliations: Zibo Center for Disease Control and

Prevention, Zibo City, China (L. Wang, T. Wang, F. Cui, S.-Y. Zhai,

L. Zhang, S.-X. Yang); Shandong Province Center for Disease

Control and Prevention, Jinan City, China (Z.-Q. Wang);

Shandong University, Jinan (X.-J. Yu); University of Texas Medical

Branch, Galveston, Texas, USA (X.-J. Yu)

DOI: http://dx.doi.org/10.3201/eid2202.151516 in patients' serum with an ELISA kit (Beijing Wantai, Beijing, China).

During 2006-2014, a total of 782 HFRS cases were reported in Zibo City; 9 (1.2\%) persons died. The incidence rate of HFRS varied from 0.74 to 3.65 per 100,000 persons annually (average 1.96 cases $/ 100,000$ persons) (Table). HFRS patients were from rural areas in all 9 districts and counties in Zibo City, but most (73.3\%) were from hilled areas in the central and southern parts of the city, including Yiyuan County (278 cases), Zichun District (161 cases), and Boshan District (134 cases) (Figure 1). During 2006-2010, HFRS patients were predominantly from Zichuan and Boshan Districts (51.8\%) in the central part of the city. During 2011-2014, Yiyuan County became the major source of HFRS cases, accounting for $51.3 \%$ of all cases during this period.

HFRS cases peaked twice each year. The spring peak occurred during March-May, and the fall/winter peak occurred during October-December (Figure 2). Serum samples from 36 patients from 2006 through 2008 were typed by focus reduction neutralization test with Hantaan virus (HTNV) strain 76-128 and Seoul virus (SEOV) strain UR in Vero cells. Reducing $80 \%$ of plaques was considered a positive result (7). Focus reduction neutralization test showed that $93.4 \%(15 / 16)$ patients from the spring peak were infected with SEOV, and $85 \%$ (17/20) from the fall/ winter peak were infected with HTNV.

The incidence for the fall/winter peak was higher than that for the spring peak for the entire city (Figures 1,2). However, the seasonal distribution of HFRS cases varied from place to place. For example, Yiyuan County and Boshan District had a substantially higher fall/winter peak of HFRS but did not have an obvious spring peak, whereas in Zichuan District, the spring peak was higher than the fall/winter peak. The number of HFRS cases in the spring peak and fall/winter peak in the 3 high-incidence areas differed significantly $\left(\chi^{2}=74.40, \mathrm{p}<0.0001\right)$.

The seasonal distribution of HFRS also varied in different years (Table; Figure 1, 2). In 2006, the spring peak was higher than the fall/winter peak, and $41.0 \%(64 / 156)$ of cases reported during that year occurred during MarchMay. In 2012, one primary peak occurred in which $73.6 \%$ $(67 / 91)$ of cases occurred during the winter. In 2007 and 2010 , the peak was not obvious in the spring or the fall. In 2009 and 2014, the spring peak was predominant, and the fall/winter peak was not obvious. In 2008, 2011, and 2013, the epidemic mainly occurred in the fall/winter, when incidence was much higher than for the spring peak. 


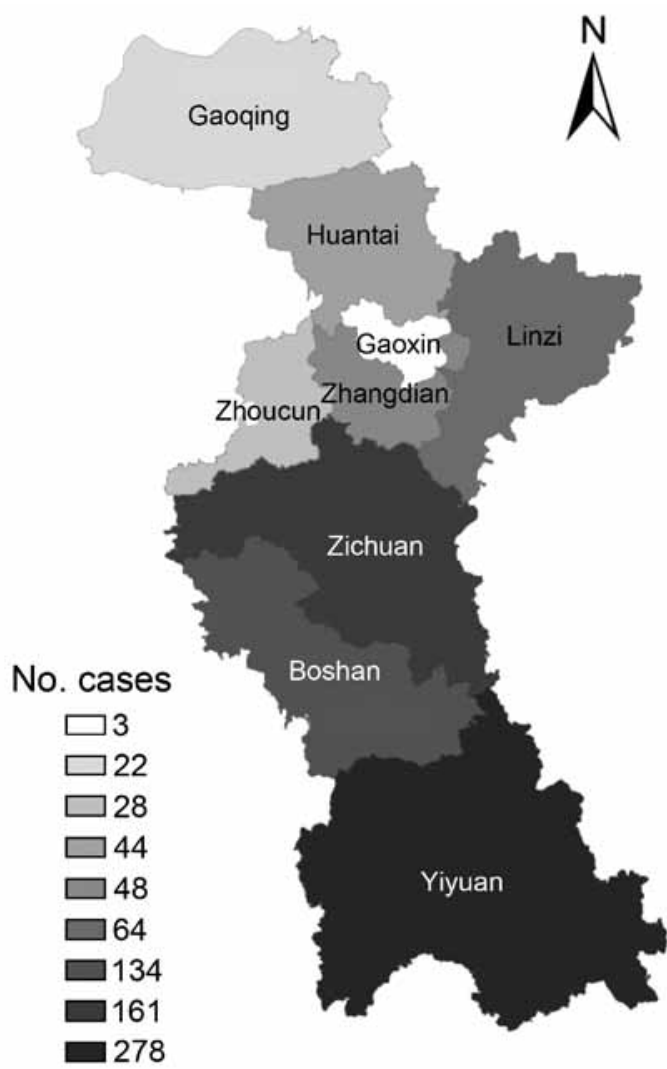

Figure 1. Geographic distribution of cases of hemorrhagic fever with renal syndrome among districts and counties, Zibo City, China, 2006-2014.

The number of HFRS cases differed significantly between the spring season and fall season each year $\left(\chi^{2}=38.01\right.$, $\mathrm{p}<0.0001)$.

Ages of the 782 HFRS patients ranged from 7 to 85 years (median 46 years). Most (73.8\%) patients were 30 65 years of age, and more than half $(51.4 \%)$ were $40-50$ years of age. Most (68.4\%) patients were farmers, and most $(87.6 \%, 685 / 782)$ lived in the countryside. The male:female ratio was 2.2:1.

Of the 9 persons who died, 4 died in October, 3 died in November, and 1 each died in August and September. Five

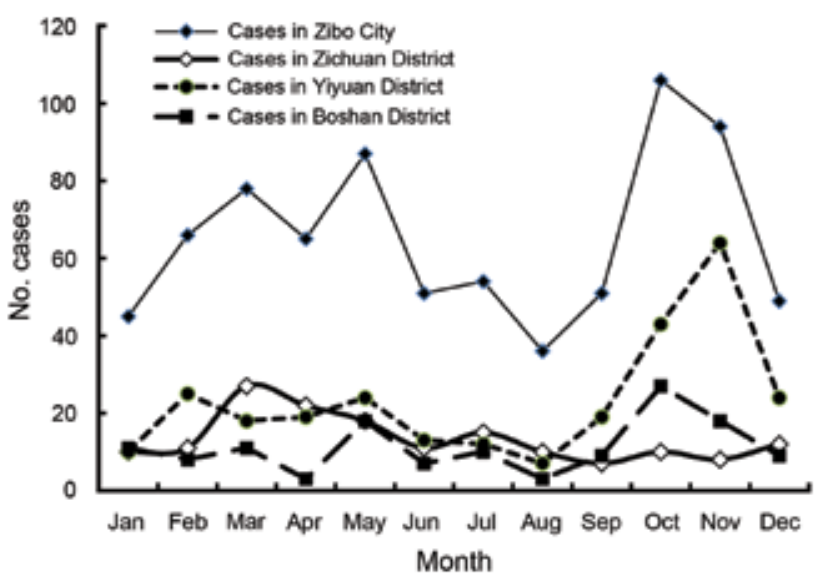

Figure 2. Monthly distribution of cases of hemorrhagic fever with renal syndrome, Zibo City, China, 2006-2014.

decedents were male. Decedent ages ranged from 33 to 60 years; 3 were $30-49$ years of age, and 6 were $50-59$ years of age. HFRS was diagnosed 3-8 days (average 5.3 days) after illness onset. The patients died 4-8 days (average 5.9 days) after illness onset.

During 2006-2014, we trapped 559 rodents with 21,384 snap-traps in residential area (inside and outside farmhouse) and in fields. The rodents' lungs were tested for hantavirus antigen with monoclonal antibody (Shanghai Institute of Biologic Products, Shanghai, China) by direct immunofluorescence assay $(8,9)$. We detected hantavirus antigens in $29(5.2 \%)$ rodents. In residential areas, the brown rat (Rattus norvegicus) was the predominant rodent species and had the highest infection rate $(5.3 \%, 21 / 393)$. The house mouse (Mus musculus) was next in both population and infection rate $(2.4 \%, 1 / 42)$. Other residential rodents were all negative for hantavirus, including 16 Chinese hamsters (Cricetulus griseus) and 1 striped field mouse (Apodemus agrarius). Among field rodents, the striped field mouse was the most common species and had the highest hantavirus infection rate $(11.9 \%, 7 / 59)$. Other field rodent species were all negative to hantavirus, including 27 brown rats, 18 buff-breasted rats (R. flavipectus), and 3 house mice. Infection rates for the striped field mouse, brown rat, and house

\begin{tabular}{lccccc}
\hline \multicolumn{6}{l}{ Table. Characteristics of hemorrhagic fever with renal syndrome, Zibo City, China, 2006-2014 } \\
\hline \\
Year & No. cases & $\begin{array}{r}\text { Incidence rate, } \\
\text { cases/100,000 population }\end{array}$ & No. deaths (\%) & Cases in spring, no. (\%) & Cases in fall/winter, no. (\%) \\
\hline 2006 & 156 & 3.65 & 0 & $64(41.0)$ & $24(15.4)$ \\
2007 & 90 & 2.10 & 0 & $31(34.4)$ & $24(26.7)$ \\
2008 & 69 & 1.60 & $1(1.45)$ & $19(27.5)$ & $29(42.0)$ \\
2009 & 35 & 0.81 & $1(2.86)$ & $14(40)$ & $5(14.3)$ \\
2010 & 32 & 0.74 & $1(3.13)$ & $6(18.8)$ & $7(21.9)$ \\
2011 & 42 & 0.93 & 0 & $5(11.9)$ & $18(42.9)$ \\
2012 & 91 & 2.00 & $2(1.40)$ & $12(13.2)$ & $67(73.6)$ \\
2013 & 136 & 2.97 & 0 & $35(25.7)$ & $45(33.1)$ \\
2014 & 131 & 2.85 & $9(1.15)$ & $44(33.6)$ & $30(22.9)$ \\
Total & 782 & 1.96 & & $230(29.4)$ & $249(31.8)$ \\
\hline
\end{tabular}


mouse did not differ significantly $\left(\chi^{2}=5.50, p=0.06\right)$. The hantavirus antigen-positive rodents were further tested by reverse transcription PCR amplification of hantavirus M segment (10) from lung tissue, and 19 rodents were PCR positive. BLAST analysis (http://blast.ncbi.nlm.nih.gov/ Blast.cgi) indicated that 13 sequences belonged to SEOV and 6 belonged to HTNV. SEOV-positive rodents were all brown rats from residential areas, and HTNV-positive rodents were all striped field mice from field areas. The reverse transcription PCR-positive rate was 3.3\% (13/393) for SEOV in brown rats from residential areas and $10.2 \%$ (6/59) for HTNV from field areas.

\section{Conclusions}

HFRS consistently occurred in Zibo City and peaked twice: in the spring and fall/winter. The spring peak was caused mainly by SEOV and the fall/winter peak mainly by HTNV. The major animal host of HTNV was the striped field mouse, and the major animal hosts of SEOV were the residential brown rat and house mouse. The house mouse is not considered the animal host of HTNV or SEOV, but this study and recent studies (11-13) have detected both HTNV and SEOV in this rodent. The role of the house mouse as the animal host of HTNV and SEOV needs further investigation.

This study was supported by grants from Shandong Province Science and Technology Development Program (2014GSF121004), National Natural Science Funds of China (31570167, 81401368), National Natural Science Foundation of China (grant no. 81102171), and Shandong Medical Science and Technology Development Program (grant no. 2011HZ055).

Dr. Ling Wang is a scientist in the Infectious Disease Department in Zibo Center for Disease Control and Prevention, Zibo City, Shandong Province, China, Her research interests include surveillance of hantavirus and emerging viral pathogens.

\section{References}

1. Meyer BJ, Schmaljohn CS. Persistent hantavirus infections: characteristics and mechanisms. Trends Microbiol. 2000;8:61-7. http://dx.doi.org/10.1016/S0966-842X(99)01658-3

2. Schmaljohn C, Hjelle B. Hantaviruses: a global disease problem. Emerg Infect Dis. 1997;3:95-104. http://dx.doi.org/10.3201/ eid0302.970202

3. McCaughey C, Hart CA. Hantaviruses. J Med Microbiol. 2000;49:587-99. http://dx.doi.org/10.1099/0022-1317-49-7-587

4. Zhang S, Wang S, Yin W, Liang M, Li J, Zhang Q, et al. Epidemic characteristics of hemorrhagic fever with renal syndrome in China, 2006-2012. BMC Infect Dis. 2014;14:384. http://dx.doi.org/ 10.1186/1471-2334-14-384

5. Wang ZQ, Wang YL, Zhao L. Surveillance and analysis on HFRS in Shandong Province from 1995 to 2000. Chin J Publ Health. 2002;18:68-70.

6. Cui F, Wang T, Wang L, Yang S, Zhang L, Cao H, et al. Spatial analysis of hemorrhagic fever with renal syndrome in Zibo City, China, 2009-2012. PLoS ONE. 2013;8:e67490. http://dx.doi.org/ 10.1371/journal.pone.0067490

7. Yiao XJ, Yu YX, An QL. Semimicro plaque assay for measuring virus titer and detecting specific neutralizing antibody of epidemic hemorrhagic fever. Chin J Virol. 1988;4:347-51.

8. Lee HW, Lee PW, Johnson KM. Isolation of the etiologic agent of Korean hemorrhagic fever. J Infect Dis. 1978;137:298-308. http://dx.doi.org/10.1093/infdis/137.3.298

9. Song G, Chen HX: Prevention and control of epidemic hemorrhagic fever. Beijing: People's Health Publishing House; 1998.

10. Wang SW, Hang CS, Wang H. Xie YX, Ma BJ, Genotype and clade distribution of hantaviruses in China [in Chinese]. Chin J Virol. 2002;18:211-6.

11. Fang LZ, Zhao L, Wen HL, Zhang ZT, Liu JW, He ST. Reservoir host expansion of hantavirus, China. Emerg Infect Dis. 2015;21:170-1. http://dx.doi.org/10.3201/eid2101.140960

12. Zuo SQ, Zhang PH, Jiang JF, Zhan L, Wu XM, Zhao WJ. Seoul virus in patients and rodents from Beijing, China. Am J Trop Med Hyg. 2008;78:833-7.

13. Sun L, Zhang YZ, Li LH, Zhang YP, Zhang AM, Hao ZY. Genetics subtypes and distribution of Seoul virus in Henan. Zhonghua Liu Xing Bing Xue Za Zhi. 2005;26:578-82.

Address for correspondence: Xue-Jie Yu, Department of Pathology, University of Texas Medical Branch, Galveston, TX 77555-0609, USA; email: yuxuejie@sdu.edu.cn

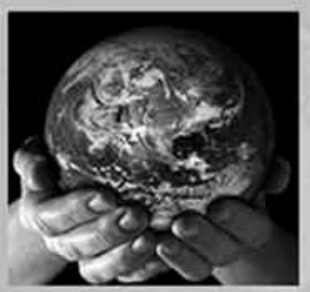

\title{
Thank You EID Reviewers
}

\section{We couldn't do it without your support.}

\begin{abstract}
All articles published in the Emerging Infectious Diseases journal are peer-reviewed by volunteers from around the globe, enabling us to bring you high-quality content about new and emerging infectious diseases and trends world-wide. To see a list of reviewers who contributed to articles published in 2015 , visit:
\end{abstract}

http://wwwnc.cdc.gov/eid/pages/reviewers.htm 